\title{
Benefits of Utilizing Pharmacy Learners in an Inpatient Anticoagulation Education Service
}

Carrie Baker, PharmD, MBA, BCPS ${ }^{1}$; Emily Ghassemi, PharmD, MSCR, BCACP, CDCES, CPP ${ }^{2}$; Riley Bowers, PharmD, BCCP, BCPS ${ }^{1}$

${ }^{1}$ Cape Fear Valley Medical Center, Campbell University College of Pharmacy \& Health Sciences

${ }^{2}$ Campbell University College of Pharmacy \& Health Sciences, SRAHEC Family Medical Center

\begin{abstract}
Objective. The 2019 Hospital National Patient Safety Goal 03.05 .01 indicates education regarding anticoagulant therapy should be provided to patients and families. Previous studies assessing pharmacist and pharmacy student involvement in oral anticoagulation (OAC) education services have focused on patient-related outcomes, with limited emphasis on the additional benefit to the student. The purpose of this study was to assess the benefit of pharmacy student involvement in anticoagulation education services both clinically and through their perceptions of participating in the service.

Methods. This study assessed students' knowledge and perceptions of providing OAC education before and after a 1-month learning experience, where students provided counseling 2-3 days per week. The primary endpoint was comparing each student's pre-and postrotation OAC education knowledge assessments. Secondary endpoints included percentage change in knowledge between the prospective cohort and a historical comparator group, perceived benefit of student participation in the service, percentage of patients able to recall counseling, and number of interventions made related to OAC therapy.

Results. A total of 35 pharmacy students were included in this prospective study with 277 patients receiving counseling from June 2020 through March 2021. A total of 32 pharmacy students had assessment data available for retrospective comparison. The mean pharmacy student score within the prospective cohort improved significantly (21.5\%) between the pre-assessment and postassessment. Change in pre-and post-rotation knowledge assessment was also significantly higher in the prospective cohort compared to the retrospective group. Additionally, students gained confidence in their counseling abilities and OAC knowledge through participation.

Conclusion. Pharmacy student participation within anticoagulation education services significantly improved student knowledge and confidence in their counseling abilities. It also allowed for over 70\% of patients to successfully recall pertinent information about their anticoagulants over a week later and improved patient care through dosing interventions.
\end{abstract}

Keywords: Counseling, Patient Care, Anticoagulants, Experiential Education

\section{BACKGROUND}

The 2019 Hospital National Patient Safety Goal (NSPG) 03.05.01 is to reduce the likelihood of patient harm associated with the use of anticoagulant therapy by ensuring appropriate protocols are in place within hospitals for the initiation and monitoring of patients receiving anticoagulation therapy. ${ }^{1}$ NSPG. 03.05.01 also indicates that education regarding anticoagulant therapy should be provided to patients and families, and include the importance of follow-up monitoring, compliance, drug-food interactions, drug-drug interactions, and potential adverse drug reactions. ${ }^{1}$ This requirement for face- to- face education by a trained professional is often fulfilled by nurses and other clinicians within the hospital system, however it creates opportunity for pharmacists and pharmacy students to provide additional direct patient care. ${ }^{1}$ Pharmacists, as the drug therapy experts, are in a position to facilitate this education and ensure understanding before the patient leaves the inpatient setting.

Corresponding author: Carrie Baker, PharmD, MBA, BCPS Cape Fear Valley Medical Center

Campbell University College of Pharmacy \& Health Sciences

Email: cnbaker0830@email.campbell.edu
It is widely accepted that utilizing pharmacists to manage the monitoring and dosing of anticoagulants in both inpatient and outpatient settings has allowed more patients to achieve a balance between therapeutic anticoagulation and bleeding risk. $^{2}$ While several studies have examined pharmacy student involvement in patient counseling services, the impact of participation in patient education on student knowledge and perceptions remains underexplored..$^{2-6}$

The majority of studies available concerning pharmacist and pharmacy student involvement in inpatient anticoagulation education focus on warfarin with less emphasis on direct oral anticoagulants (DOACs). Wilhelm and colleagues specifically looked at the impact of an anticoagulation service using pharmacy students and residents on rate of education completed and effect of providing education on overall and anticoagulation-associated readmission rates. ${ }^{2}$ Students and residents provided services while on rotation three days per week for five hours each day for patients receiving low molecular weight heparin, fondaparinux, or warfarin. ${ }^{2}$ After five months, there was a significant increase in the number of patients educated during the initial five months of service implementation at $59.2 \%$, compared to a prespecified five month timeframe prior to implementation where $39.1 \%$ of patients were educated $(p<0.001) .^{2}$ All-cause readmission rates as well as anticoagulation related readmissions for those patients who received anticoagulation education were also 
lower at 60 days. $^{2}$ A study conducted in China by Zdyb and colleagues examined the impact of pharmacist-led education and follow-up services at a tertiary medical center. ${ }^{3}$ This study found that at 30 days, those patients who received pharmacist led-education through one-on-one education sessions and a patient education booklet prior to discharge had a better knowledge of warfarin as indicated by answering more survey questions correctly at 30 days post-discharge. ${ }^{3}$ Similarly, a 2018 study conducted by Kim and colleagues examined the utilization of student pharmacists in DOAC monitoring. ${ }^{4}$ Student led interventions in this study included adherence education, medication access, monitoring recommendations, and alternative anticoagulation recommendations. ${ }^{4}$ This study found that student pharmacists can help ensure medication safety and effective use of DOACs, however similar to the other studies, it did not assess the impact participation had on student pharmacists. ${ }^{4}$

A study by Dunkley and colleagues also examined the utilization of student pharmacists to provide medication education on anticoagulants, inhalers, insulin, and naloxone. This study utilized 10 student pharmacists and found participants felt sufficiently to exceedingly prepared to perform medication education. ${ }^{5}$ Overall rates of patient education were increased from $79 \%$ to $86 \%(p=0.006) .{ }^{5}$ However, this study did not assess patient understanding or satisfaction with education provided by student pharmacists. ${ }^{5}$ An additional study by Slater and colleagues examined student pharmacists as interprofessional educators for student nurses and student physicians on insulin management. ${ }^{6}$ This study indicated that over $90 \%$ of student nurses and student physicians found pharmacists to provide added value to the medical team, and $95 \%$ of surveyed learners also indicated improved confidence in making clinical decisions regarding insulin therapy following student pharmacist education. ${ }^{6}$ This study demonstrates the usefulness of student pharmacists as clinical educators, although not directly assessing their impact as it relates to anticoagulation.

While studies have indicated that pharmacists and pharmacy students are effective at providing anticoagulation education services, examining the impact of participation on pharmacy student knowledge and perceived benefits are under-explored. Thus, the purpose of this study was to assess the benefit of pharmacy student involvement in anticoagulation education services both clinically and through their perceptions of participating in the service.

\section{METHODS}

Study Design. This was a prospective cohort study with a historical comparator assessing the impact of pharmacy student participation in an OAC counseling service on their knowledge, confidence, and perception towards providing counseling. Students in the 2020-2021 cohort served as their own controls for knowledge assessments and perceptions, and were also compared to a historical comparator group who completed pre/post knowledge assessments only prior to implementation of the formal anticoagulation education service.

In Spring 2020, an education service for patients being discharged on a new OAC was implemented to improve patient care and address the 2019 Hospital NSPG 03.05.01. ${ }^{1}$ Patients requiring $O A C$ education were identified through the use of a daily report conducted within the electronic medical record (EMR). Education was then provided by students in person to patients prior discharge both verbally and with written materials. Due to part of this study being conducted during the COVID-19 pandemic, patients who were positive for COVID-19 were counseled over the phone instead of in person. All other patients continued to receive in person counseling. Once education was completed for each patient, interventions were documented within the EMR. One-week post-discharge phone calls were completed by pharmacists and pharmacy students to assess patient knowledge of their OAC and memory of the counseling session itself. Follow-up call questions were modified from the anticoagulation knowledge tool, which is a validated tool allowing for open ended patient responses related to pertinent medication administration and safety information patients should be able to recall. ${ }^{7}$ This study design was reviewed and approved by the Cape Fear Valley IRB prior to initiation.

Following a one-month pilot by pharmacists and pharmacy residents in May 2020, students were added to the service as part of every rotation at the medical center to expand pharmacy's clinical impact on patient care. Students from June 2020 through March 2021 participated in the service. Those students with multiple rotations at the medical center were included in the counseling service on each learning experience, however the pre- and pos- knowledge assessments as well as perception questionnaires were only administered during each student's initial month participating in the service. Students received a 30-question multiple choice pre-assessment to objectively assess their knowledge of OACs prior to rotation as well as a survey assessing their perceptions associated with counseling patients and confidence in performing counseling prior to rotation. This survey used a 5-point Likert scale with answers ranging from extremely knowledgeable to not knowledgeable at all. Perception surveys were designed based on competencies highlighted by the pharmacist patient care process (PPCP) with respect to assessing, planning, and implementation. To ensure consistency and clarity of perception surveys these were calibrated using first year pharmacy residents. Assessment questions were also calibrated utilizing pharmacy residents with questions complied from previous pharmacy student didactic examinations and created by the research team.

During the orientation portion of rotation, students underwent a three-hour training education session where pertinent information concerning each of the OACs and proper patient counseling techniques were reviewed and modeled. Students 
on an advanced practice pharmacy experience (APPE) were accompanied by a pharmacist to receive a sign-off of adequate knowledge to perform counseling independently. Introductory pharmacy practice experience (IPPE) students were accompanied by a pharmacist or APPE student during all patient counseling sessions. Students participated in counseling two to three days per week during their month-long rotations.

Following each rotation students were again administered the multiple-choice assessment questions to determine the impact counseling had on each student's knowledge of OACs. The perception survey was administered as well to assess for changes in student perceptions and confidence through participation in the education service. In addition to the initial perception questions, the follow-up perception survey also included 10 questions related to student perceptions of patient benefit from counseling services. This was assessed using a 7point Likert scale with answers ranging from strongly agree to strongly disagree. The impact of time saved through utilization of students was also assessed on a monthly basis through documentation in patient interventions noting the time students spent counseling each patient. An overview of the study design can be seen in Figure 1.

Prior to implementation of the service, from November 2019 through April 2020, pharmacy students completing rotations at the medical center also completed the pre-rotation and postrotation knowledge assessments and received similar instruction on OAC but did not participate in a continuous education process. No surveys were administered to assess the perceptions of this historical comparator group. The use of this historical comparator group was included to allow for a better comparison of the impact of the actual service versus what was being learned on each student's individual rotation experience. The assessment the historical comparator group completed was identical to the prospective cohort.

Study Objectives and Endpoints. The primary endpoint was the mean percentage difference in pharmacy student pre- and post- OAC education knowledge assessments in the 2020-2021 student cohort. Percentage change in knowledge from the preand post- OAC education knowledge assessment was then compared between the prospective cohort and historical comparator that did not participate in the counseling service as the first secondary endpoint. Other secondary endpoints from the prospective cohort only related to student perceptions included perceived benefit of participation in OAC education determined through surveys assessing confidence and value of the activity. Secondary endpoints related to hospital outcomes and patient related outcomes included the value of education completed by pharmacy students as measured through time saved by utilizing students to provide counseling, percentage of patients able to recall the education session, indication, signs and symptoms of bleeding, and medication they were receiving at hospital discharge, and the number of interventions made related to inappropriate OAC therapy.
Study Population. Pharmacy students from multiple colleges of pharmacy in their second through fourth years of pharmacy school who had rotations at Cape Fear Valley Medical Center from June 2020 through March 2021, or June 2019 through April 2020 for the historical comparator, were included in this study. IPPE students who were included in the historical comparator group were not included in the prospective group analysis if they had subsequent APPE rotations at the facility. These students were allowed to participate in the service.

Statistical Analysis. Differences in the primary endpoint were assessed using a paired T-test. The secondary endpoint of change in knowledge assessment scores between groups was analyzed using a Student T-test, while the secondary endpoint of perceived benefit of participation in the OAC education service was assessed using a Wilcoxon Signed Rank Test. All remaining secondary endpoints were analyzed using descriptive statistics. Statistical analyses were performed using JMP14-PRO (SAS, Cary, NC).

\section{RESULTS}

A total of 35 students were included in this study. Of those 35 students, 24 were on their APPEs, while 11 were on their IPPEs. The most common learning experience was internal medicine (42.9\%), followed by introduction to hospital (22.9\%). These characteristics were similar to those in the retrospective comparator. Out of 32 students, 23 were on APPEs, and 9 were on IPPEs. Other relevant baseline characteristics are summarized in Table 1.

The primary endpoint of mean percentage difference in student knowledge between pre- and post- OAC education assessments significantly improved by $21.5 \%$, from $48.7 \%$ to $70.2 \%$ respectively $(95 \% \mathrm{Cl} 0.18$ to $0.25, \mathrm{p}<0.0001)$. The prospective group also demonstrated a greater change in assessment scores compared to the retrospective comparator that did not participate in the service, with $+21.5 \%$ versus $+8.6 \%$ respectively $(95 \% \mathrm{Cl} 0.86$ to $3.11, \mathrm{p}<0.0001)$ (Table 2$)$. Within the prospective group only, secondary endpoints related to changes in student perceptions based on participation in the service were also all statistically significant $(p<0.0001)$. These included how students perceived their ability to explain mechanisms of action, recommend alternative anticoagulants, answer patient questions related to OAC, and explain signs and symptoms of bleeding (Table 3). Students also reported their perceptions of patient benefit from the counseling service at the end of each learning experience (Table 4). Over $65 \%$ of students felt patients benefited from counseling while inpatient and also believed the utilization of teach back methods and questions were effective for patient knowledge retention. Furthermore, over $80 \%$ believed that participation in the service augmented their OAC knowledge and confidence and allowed them to feel they were improving patient care. 
Secondary endpoints focused on patient knowledge retention reflected students' perceptions of patient benefit. Of the 277 patients receiving counseling, 124 patients (45\%) were available for follow-up call surveys one week after discharge. During these surveys, $90.3 \%$ of patients were able to recall their medication name and indication, $73.4 \%$ recalled the signs and symptoms of bleeding and $87.9 \%$ found the counseling sessions to be useful (Table 5). Additionally, 40 interventions were made related to inappropriate OAC therapy. Of the 40 interventions, 33 were related to inappropriate dosing, 4 were related to duplicate therapies, and 3 were related to a change in OAC. Finally, the average time spent counseling was 15 minutes per patient which equated to approximately 70 hours of total time saved for pharmacists.

\section{DISCUSSION}

In this prospective cohort study, we were able to demonstrate benefit provided to students, patients, and the health system. A significant difference was seen between student pre- and post- OAC knowledge assessments, and student perceptions were also influenced through participation in the OAC education service. Additionally, knowledge of OAC was increased for those students who formally participated in the OAC education service compared to those who received OAC experience through their rotation alone. Students also perceived patients as deriving benefit from the OAC counseling. This study highlights the need for both didactic and clinical components in experiential learning, and the benefit of student involvement in education services to both students and patients. The importance of integrating opportunities for students to apply the PPCP within an institutional or inpatient setting was previously highlighted in a study by Taylor and collegues. ${ }^{8}$ This study indicated students often have a harder time identifying activities associated with collection and implementation within inpatient settings and challenged faculty to provide early opportunities for students to apply the PPCP in this setting. ${ }^{8}$ The implementation of an OAC education service facilitated this clinical application of didactic skills including the collection of information, creation of therapeutic plans, and implementation of counseling skills in a setting where these opportunities are often lacking compared to ambulatory care settings.

While the majority of included students were in their final year of pharmacy school and on internal medicine rotations, the benefit derived from participation in the service was present for students on all experiences based on aggregate data collected during this study. Furthermore, the benefit in change in knowledge was consistent throughout the academic year indicating that even as fourth year students progressed through their final year, this service was able to continually augment their understanding and clinical skillset. While the mean postrotation knowledge assessment score of $70.2 \%$ in the prospective cohort, and $62.2 \%$ in the retrospective comparator may be perceived as below average, the assessment was designed to be challenging with questions written at or above the level required for students to pass the pharmacy licensing exam. Utilizing an assessment at this level not only challenged students during their rotations, but also afforded them an additional opportunity to prepare for their licensing examination following completion of pharmacy school. In addition, the mean final assessment score was not only better compared to students from the previous year who did not participate in the OAC education service, but the increase in knowledge scores was nearly $13 \%$ higher in those who participated in the service.

Through the inclusion of a post-service questionnaire with questions designed to assess student's viewpoint on the benefit of counseling to the patient as well as allow for student selfreflection, this study was able to assess activity value. The majority of students revealed agreement or strong agreement with the benefit of this service to both patients and themselves, indicating that active participation in the learning process not only increased their confidence and allowed them to develop a process for their future careers, but also increases benefit to the patient. These results are in agreement with results found by Dunkley and colleagues where ten students were utilized to provide medication education following a training program, and of the seven students surveyed all felt sufficiently to extremely prepared to provide medication education. ${ }^{5}$ While our study affirms the positive impact of student participation in education services, we did have several differences from Dunkley and colleagues. First, we examined student knowledge and perceptions over a one-month timeframe as compared to a year and included students on their IPPE and APPE learning experiences with two of those students only having completed their first year of pharmacy school prior to participation in the OAC education service. Finally, we were also able to assess patient understanding and satisfaction of student provided education through post-discharge follow up calls and found the perceptions of students and patients to be similarly aligned.

Of the counseled patients, $45 \%$ participated in follow up calls with approximately $75 \%$ of patients correctly answering questions related to their OAC. These results indicate the importance of discharge counseling and medication review with patients prior to discharge. These outcomes align with those found by Zdyb and colleagues where patients who received one-on-one education had improved knowledge of their OAC. ${ }^{3}$ The effectiveness of student pharmacists as peer educators was also previously highlighted by Slater and colleagues where student pharmacists were able to impact the knowledge and confidence of student nurses and student physicians. ${ }^{6}$ While our study addresses student pharmacists as patient educators it affirms the conclusions from Slater and colleagues with students being able to provide effective education and helping to add value to interdisciplinary medical teams through this skill. 
During follow-up calls, patients were also asked to provide any changes they wished to see to the service. Of the 124 patients who responded to the calls, three patients provided feedback on changes to the service. One patient recommended providing a phone number to the patient at the time of discharge for additional questions once they returned home, another suggested more family members be involved in counseling, and the third recommended waiting until they had been more awake and aware during their hospitalization. This patient feedback raises considerations for the current counseling service and provides a direction for future growth.

The 40 interventions made regarding inappropriate OAC therapy along with time saved to pharmacy staff and nursing regarding counseling illustrated the benefit to the health system created by utilizing students. This benefit is similar to the benefit noted by Kim and colleagues where studentpharmacists were utilized for DOAC monitoring and ensured medication safety and effective use of medications. ${ }^{4}$ However, unlike Kim and colleagues., this study also focused on the benefit students derived from participation in the service.

While this study included both IPPE and APPE students, and benefits of the education service were able to be assessed across multiple domains including the students, patients and the health system, there were some limitations. Although students on any rotation within the hospital were included, the majority of students were on internal medicine and there was no way to adjust for improvement in anticoagulation knowledge secondary to participation in this learning experience versus the anticoagulation service itself. Students were also un-blinded to the perception surveys being part of a research project, which may have altered their responses to be more favorable. Finally, those students administered the knowledge assessments in the historical cohort did not provide their perceptions of participating in counseling to provide a comparison to students who participated in the formalized service.

\section{CONCLUSION}

Pharmacy student participation within the education service improved overall student knowledge and confidence in their ability to provide counseling. This study highlights the practicality of integrating students into clinical workflow to improve student knowledge and confidence, as well as augmenting clinical services offered by pharmacy within an inpatient setting. Based on the results of this study, including students in other clinical activities such as medication reconciliations, device counseling, and high-risk medication counseling may serve as a future opportunity to enhance clinical skills developed in didactic settings and further improve pharmacists' impact on transitions care.
Acknowledgments: None

Funding/Support: None

Conflicts of Interest: None

\section{REFERENCES}

1. The Joint Commission. National Patient Safety Goals 2020 for the Hospital Program. The Joint Commission. Available from https://www.jointcommission.org/standards/national -patient-safety-goals/hospital-2020-national-patientsafety-goals/.

2. Wilhelm SM, Petrovitch EA. Implementation of an inpatient anticoagulation teaching service: Expanding the role of pharmacy students and residents in patient education. American Journal of HealthSystem Pharmacy. 2011;68(21):2086-2093.

3. Zdyb EG, Courtney DM, Malik S, Schmidt MJ, Lyden AE. Impact of Discharge Anticoagulation Education by Emergency Department Pharmacists at a Tertiary Academic Medical Center. Journal of Emergency Medicine. 2017;53(6):896-903.

4. Kim JJ, Hill HL, Groce JB 3rd, Granfortuna JM, Makhlouf TK. Pharmacy Student Monitoring of Direct Oral Anticoagulants. J Pharm Pract. 2018;31(5):462468.

5. Dunkley KA, Evelyn D, Timmons V, Feller TT. Implementation of a medication education training program for student pharmacists employed within an academic medical center. Am J Health Syst Pharm. 2020;77(3):206-213. doi:10.1093/ajhp/zxz309

6. Slater N, Todd A, Grimm A. Pharmacy students as educators: An interprofessional approach to insulin management education. Currents in pharmacy teaching and learning. 2020;12(6):689-693. doi:10.1016/j.cptl.2020.01.034

7. Obamiro KO, Chalmers L, Bereznicki LR. Development and Validation of an Oral Anticoagulation Knowledge Tool (AKT). PLoS One. 2016;11(6):e0158071. Published 2016 Jun 28. doi:10.1371/journal.pone.0158071

8. Taylor S, Deja E, Divine H, Laney L, Mclntosh T. Pharmacy Students' Ability to Identify the Steps of the Pharmacists' Patient Care Process During IPPE. American journal of pharmaceutical education. 2020;84(4):7453-420. doi:10.5688/ajpe7453 
Table 1. Baseline Characteristics of Pharmacy Students

\begin{tabular}{|c|c|c|}
\hline Characteristic & $\begin{array}{l}\text { Prospective Cohort: } 2020-2021, \\
\mathrm{~N}=35\end{array}$ & $\begin{array}{l}\text { Retrospective Comparator: } 2019- \\
2020, N=32\end{array}$ \\
\hline \multicolumn{3}{|l|}{ Graduation Year - n (\%) } \\
\hline 2020 & - & $23(71.9)$ \\
\hline 2021 & $24(68.6)$ & $7(21.9)$ \\
\hline 2022 & $9(25.7)$ & $2(6.3)$ \\
\hline 2023 & $2(5.7)$ & - \\
\hline \multicolumn{3}{|l|}{ Rotation Type $^{a}-\mathrm{n}(\%)$} \\
\hline Introduction to Community & $2(5.7)$ & $2(6.3)$ \\
\hline Introduction to Hospital & $9(25.7)$ & $7(21.9)$ \\
\hline Advanced Community & $3(8.6)$ & $3(9.4)$ \\
\hline Advanced Hospital & $4(11.4)$ & $3(9.4)$ \\
\hline Internal Medicine & $15(42.9)$ & $15(46.9)$ \\
\hline Elective APPEs & $2(5.7)$ & $2(6.3)$ \\
\hline \multicolumn{3}{|l|}{ Oral Anticoagulant Counseled On - n (\%) } \\
\hline Warfarin & $25(9.0)$ & - \\
\hline Apixaban & $227(81.9)$ & - \\
\hline Rivaroxaban & $25(9.0)$ & - \\
\hline
\end{tabular}

${ }^{a}$ Advanced Community, Advanced Hospital, Internal Medicine, and Elective APPES all represented APPE experiences, while Introduction to Community and Introduction to Hospital were IPPE experiences

Table 2. Knowledge Assessment Scores ${ }^{\mathrm{a}}$

\begin{tabular}{|c|c|c|}
\hline \multicolumn{3}{|l|}{ Primary Outcome ${ }^{b}-$ Prospective Cohort, $\mathrm{N}=35$} \\
\hline \multicolumn{3}{|l|}{ Knowledge Assessment Score } \\
\hline Pre-rotation & $48.7 \%$ & \\
\hline Post-rotation & $70.2 \%$ & \\
\hline Percentage difference & $+21.5 \%$ & $95 \% \mathrm{Cl} 0.18-0.25, \mathrm{p}<0.0001$ \\
\hline \multicolumn{3}{|c|}{ Secondary Outcome - Prospective Cohort vs. Retrospective Comparator ${ }^{\mathrm{c}}(\mathrm{N}=32)$} \\
\hline \multicolumn{3}{|c|}{\begin{tabular}{l|l|l} 
Knowledge Assessment Score (Retrospective) & &
\end{tabular}} \\
\hline Pre-rotation & $53.6 \%$ & \\
\hline Post-rotation & $62.2 \%$ & \\
\hline \multicolumn{3}{|c|}{ Change in Knowledge Assessment Scores Between Prospective Cohort and Comparator } \\
\hline Prospective Cohort & $+21.5 \%$ & \multirow[t]{2}{*}{$95 \% \mathrm{Cl} 0.86-3.11, \mathrm{p}<0.0001$} \\
\hline Retrospective Comparator & $+8.6 \%$ & \\
\hline
\end{tabular}


Table 3. Student Perceptions and Confidence before and after OAC service Participation ${ }^{\mathrm{a}, \mathrm{b}}$

\begin{tabular}{|l|l|l|l|}
\hline $\begin{array}{l}\text { Changes in student perception based on participation } \\
\text { in service: }\end{array}$ & $\begin{array}{l}\text { Pre-Service } \\
\text { Mean Score }\end{array}$ & $\begin{array}{l}\text { Post-Service } \\
\text { Mean Score }\end{array}$ & P-value \\
\hline Explaining the mechanism of action & $2.8 \pm 0.6$ & $4.1 \pm 0.7$ & $<0.0001$ \\
\hline Assessing appropriateness of anticoagulation therapy & $2.9 \pm 0.6$ & $3.9 \pm 0.7$ & $<0.0001$ \\
\hline Recommending alternative OAC agents & $2.6 \pm 0.7$ & $3.8 \pm 0.8$ & $<0.0001$ \\
\hline Answering patient questions concerning OAC & $2.8 \pm 0.7$ & $4.2+0.8$ & $<0.0001$ \\
\hline $\begin{array}{l}\text { Recognizing and informing patients of drug-drug } \\
\text { interactions with OAC }\end{array}$ & $2.6 \pm 0.7$ & $3.7 \pm 0.9$ & $<0.0001$ \\
\hline Explaining signs/symptoms of bleeding & $3.7 \pm 0.8$ & $4.7 \pm 0.6$ & $<0.0001$ \\
\hline
\end{tabular}

a Student perceptions were assessed using a five point Likert scale with students assessing themselves as one of the following: not knowledgeable at all( 1), slightly knowledgeable (2), moderately knowledgeable (3), very knowledgeable (4), and extremely knowledgeable (5).

${ }^{\mathrm{b}}$ A Wilcoxin Signed Rank test was utilized to compare pre- and post- OAC education service student perceptions.

Table 4. Student Perceptions of Patient Benefit from Counseling Service

\begin{tabular}{|c|c|c|c|c|}
\hline \multicolumn{5}{|c|}{ Student Perceptions of Patient Benefit from Counseling Service ${ }^{a}(\mathrm{~N}=35)$} \\
\hline & $\begin{array}{l}\text { Strongly Agree - } \\
\mathrm{n}(\%)\end{array}$ & Agree $-\mathrm{n}(\%)$ & $\begin{array}{l}\text { Some-what } \\
\text { Agree - } \mathrm{n}(\%)\end{array}$ & $\begin{array}{l}\text { Neither agree nor } \\
\text { disagree - n (\%) }\end{array}$ \\
\hline $\begin{array}{l}\text { Patient benefitted from counseling while } \\
\text { inpatient }\end{array}$ & $23(65.7)$ & $10(28.6)$ & $2(5.7)$ & 0 \\
\hline Patient given the opportunity to ask questions & $32(91.4)$ & $3(8.6)$ & 0 & 0 \\
\hline $\begin{array}{l}\text { Patient understood signs/symptoms of bleeding } \\
\text { and when to ask for help }\end{array}$ & $24(70.6)$ & $11(31.4)$ & 0 & 0 \\
\hline $\begin{array}{l}\text { Patient was provided accurate and thorough } \\
\text { answer to questions }\end{array}$ & $28(80)$ & $7(20)$ & 0 & 0 \\
\hline $\begin{array}{l}\text { Using teach-back methods and asking questions } \\
\text { related to key points was effective }\end{array}$ & $24(68.6)$ & $9(25.7)$ & $2(5.7)$ & 0 \\
\hline $\begin{array}{l}\text { Providing both verbal and paper education was } \\
\text { more effective than either alone }\end{array}$ & $30(85.7)$ & $4(11.4)$ & $1(2.9)$ & 0 \\
\hline $\begin{array}{l}\text { Participation in service improved my OAC } \\
\text { knowledge }\end{array}$ & $29(82.9)$ & $6(19.1)$ & 0 & 0 \\
\hline $\begin{array}{l}\text { Participating in service improved my confidence } \\
\text { in counseling on OAC }\end{array}$ & $31(88.6)$ & $4(11.4)$ & 0 & 0 \\
\hline $\begin{array}{l}\text { Participating in the service was a quality } \\
\text { rotation activity }\end{array}$ & $27(77.1)$ & $8(22.9)$ & 0 & 0 \\
\hline $\begin{array}{l}\text { Overall I feel I was able to improve patient care } \\
\text { through this service }\end{array}$ & $28(80)$ & $6(17.1)$ & $1(2.9)$ & 0 \\
\hline
\end{tabular}

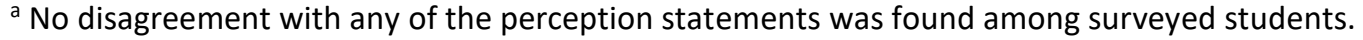


Table 5. Patient and Hospital Outcomes

\begin{tabular}{|l|l|}
\hline \multicolumn{2}{|l|}{ Additional Secondary Outcomes } \\
\hline $\begin{array}{l}\text { Percentage of patients able to recall medication and } \\
\text { indication }(\mathrm{N}=124)-\mathrm{n}(\%)\end{array}$ & $112(90.3)$ \\
\hline $\begin{array}{l}\text { Percentage of patients able to recall signs/symptoms of } \\
\text { bleeding }(\mathrm{N}=124)-\mathrm{n}(\%)\end{array}$ & $91(73.4)$ \\
\hline $\begin{array}{l}\text { Percentage of patients who found counseling useful } \\
(\mathrm{N}=124)-\mathrm{n}(\%)\end{array}$ & $109(87.9)$ \\
\hline Number of interventions related to inappropriate OAC & 40 interventions \\
\hline Average time spent performing OAC education & 15 minutes \\
\hline
\end{tabular}

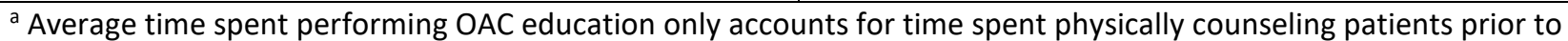
discharge and does not include time spent completing post-discharge follow up calls.

Figure 1. Overview of Study Design

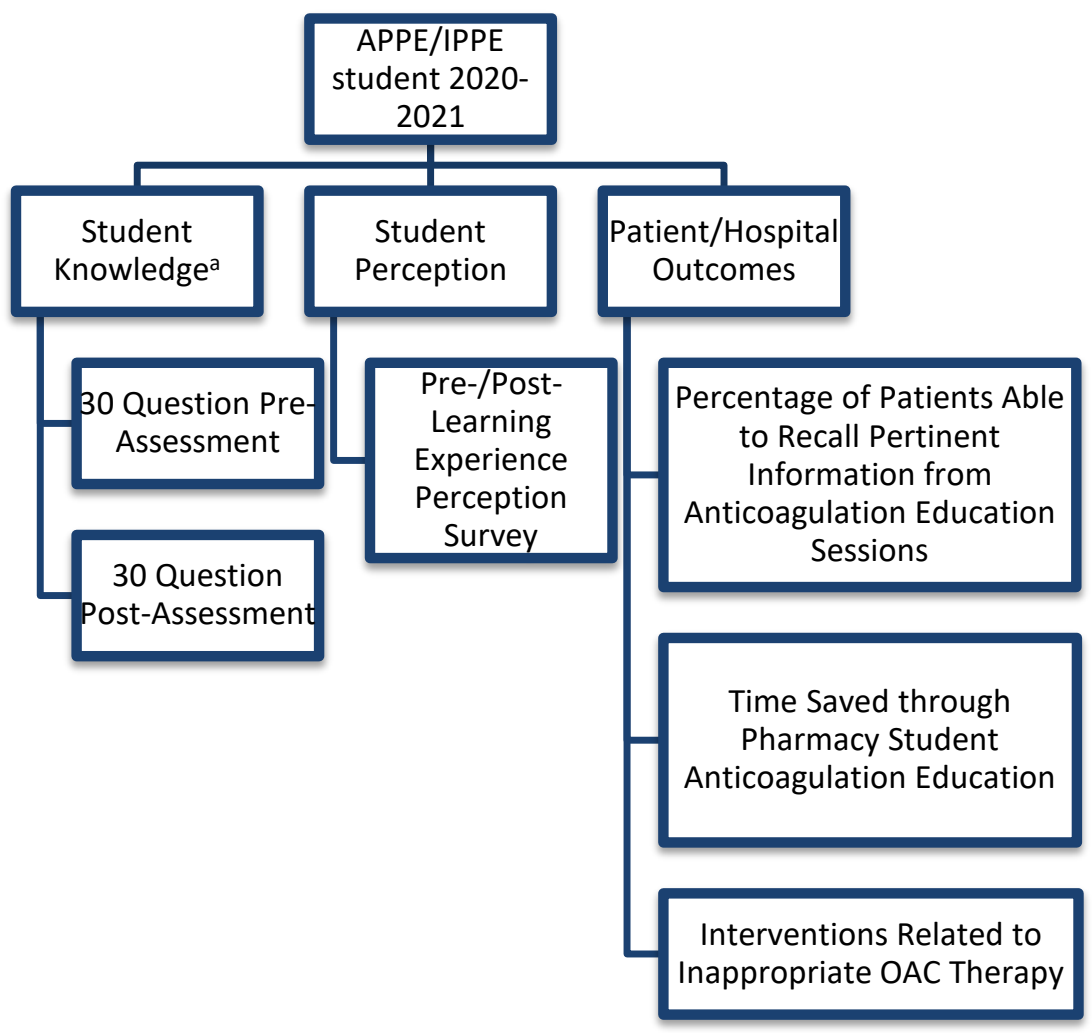

Figure 1. This study assessed student knowledge, student perceptions, and benefit to the patient as well as the health system. A series of surveys were utilized to assess student knowledge and perceptions, while follow-up phone calls were used to assess patient knowledge retention. Knowledge assessments collected prior to implementation of the oral anticoagulation service were also used to analyze the impact of the formal service on student knowledge.

a Students in the historical comparator cohort from 2019-2020 participated only in the student knowledge assessments pre- and post- learning experience, but did not participate in perception surveys completed by the prospective group. 